\title{
Author Index Vol. 23, 1995
}

Adams, J. 76 Adelson, P.D. 26 Aitken, P.A. 1 Aksaray, N. 328 Albright, A.L. 20, 82, 86 Alhan, E. 328 Altunbasak, S. 328 Argemand, E. 159 Armstrong, D. 148 Arnold, S. 68 Aronin, P.A. 7

Bakker Niezen, S.H. 270 Barquin, N. 283 Barry, M.J. 82 Bartels, R.H. 270 Baule, R. 305 Baytok, V. 328 Beals, S.P. 199 Beeler, L.M. 76 Bell, W.O. 42 Blaivas, M. 216 Boal, D. 305 Bondurant, C.P. 254 Boydston, W.R. 323 Buchbinder, B.R. 122 Bullock, P. 188 Byrne, R.W. 182

Cahan, L.D. 76 Casale, A.J. 228 Chandra Mouli, B.A. 57 Chapman, P.H. 122 Chi, C.-S. 192 Cho, D.-Y. 192 Chou, P.M. 283 Clyde, B.L. 20 Cochrane, D.D. $14,206,317$ Constantini, S. 279 Cosgrove, G.R. 122 Craft, S. 68

Das, B.S. 57 Dauser, R.C. 216 Dehner, L.P. 159 DiRocco, C. 115 
Doran, S.E. 216 Drake, J. 148

Epstein, F. 279 Evans, A. 188 Evans, D. 14

Farmer, J.-P. 93 Fasick, M.P. 82 Forte,|V. 148 Francel, P.C, 159 Friedman, H.S. 293

George, T.M. 182,236 Gerszten, P.C. 86 Gomori, J.M. 279 Grätz, K.W. 54 Greffe, B.S. 219

Harlow, C.L. 140 Haw, C.S. 206 Hayes,|E.A. 182 Heron, N. 14 Hockley, A.D. 127 Hoffman, H.J. 6,148 Hönger, M. 54 Hudgins, RJ. 323 Humphreys, R.P. 148,332 Iannelli, A. 115 Illii, O.E. 54

Janosky, J. 82 Jiang, H.J. 122 Jimenez, D.F. 254 Joganic, E. 199 Johnson,|D.L. 305 Johnson, M.C. 273

Kaufman, B.A. 159, 171 Kerby, T. 293 Kerrick, R. 7 Kestle, J.R.W. 14,206 Kirk, E. 64 Klauber, M.R. 34 Kolodny, E.H. 225 
Lam, C.H. 93 Leshinsky, E. 225 Linden, R.D. 228 Luerssen, T.G. 34

Macfarlane, R. 148 McGavran, L. 219 McLone, D.G. 182,236,311 Malluci, Cl 127 Manwaring, K.H. 199 Manz, HJJ. 97 Marchese, E. 115 Mastromateo, J. 1 Matsumura, A. 166 Meagher-Villemure, K. 93 Meguro, K. 166 Menezes, A.H. 101,260 Moghrabi, A. 293 Mohanty, A. 57 Montes, J.L. 93 Moran, C.J. 171 Moss, S.D. 199 Muhlbauer, M.S. 64

Nakada, Y. 166 Narushima, K. 166 Nazar, G.B. 228 Noetzel, M. 68 Nose, T. 166

Odell, J.M. 273

Pang, D. 227 Park, T.S. 68, 159,171 Partington, M.D. 140,219,311 Perry,|J. 76 Phillips,|J. 148 Piatt, J.H., Jr. 133 Pople, I.K. 64 Posnick, J. 148 Prall, J.A. 219

Rao, S. 279 Renzulli, P. 54 Reyes-Mugica, M. 283 Roberts, J.G. 228 
Roberts, R.L. 159 Robinson, L. 188 Rotteveel, J.J. 270 Rutka, J.T. 148

Sanford, R.A. 64 Sastry Kolluri, V.R. 57 Satish, S. 57 Sato, O. 246 Sato, Y. 101 Schatz, J. 68 Schellinger, D. 97 Scott,|R.M. 26 Sgouros, S. 127 Siegal, T. 279 Spettell, CM. 299 Stehbens, W.E. 97 Stein, S.C. 299 Steinbok, P. 14,206,317 Subbakrishna, D.K. 57

Takei, F. 246 Tan, X. 283 Thieme, G.A. 140 Thijssen, H.O. 270 Thomas,|N.H. 188 Tien, R, D. 293 Tomita, T. 283 Tsurushima, H. 166

Uszinski, R. 97

Vieco, P.T. 1

Wald, S.L. 1 Walsh,|A.R. 127 Wang, Y.-C. 192 White,|D.A. 68 Wilson,|J.T. 1

Yasuda, T. 283 Yonekawa, Y. 54 Yuksel, B. 328

Zelnik, N. 225 